\title{
Stability and Hopf Bifurcation Analysis of a Gene Expression Model with Diffusion and Time Delay
}

\author{
Yahong Peng ${ }^{1}$ and Tonghua Zhang ${ }^{2}$ \\ ${ }^{1}$ Department of Mathematics, Donghua University, Shanghai 200051, China \\ ${ }^{2}$ Department of Mathematics, Swinburne University of Technology, Melbourne, VIC 3122, Australia
}

Correspondence should be addressed to Yahong Peng; pengyh.mail@163.com

Received 24 February 2014; Accepted 13 March 2014; Published 14 April 2014

Academic Editor: Weiming Wang

Copyright (c) 2014 Y. Peng and T. Zhang. This is an open access article distributed under the Creative Commons Attribution License, which permits unrestricted use, distribution, and reproduction in any medium, provided the original work is properly cited.

We consider a model for gene expression with one or two time delays and diffusion. The local stability and delay-induced Hopf bifurcation are investigated. We also derive the formulas determining the direction and the stability of Hopf bifurcations by calculating the normal form on the center manifold.

\section{Introduction}

The study on dynamics of a biological model is one of the dominant subjects in mathematical biology due to its universal existence and importance. In this paper, we consider a mathematical model of intracellular regulatory system which began with the work of Goodwin [1]. Since then, many researchers developed this work [2-5]. However, it is Monk who developed the first mathematical model for the Hesl system and validated it with biological data [6]. In order to describe the intracellular process more precisely, he introduced time delays to account for the processes of transcription and translation. If we denote by $M(t)$ and $P(t)$ the concentrations of Hes1 mRNA and Hes1 protein, respectively, the basic reaction kinetics for this system can be expressed in the form of

$$
\begin{gathered}
\frac{d M(t)}{d t}=f\left(P\left(t-\tau_{m}\right)\right)-c M(t), \\
\frac{d P(t)}{d t}=a M\left(t-\tau_{p}\right)-b P(t),
\end{gathered}
$$

where $f(P)=\alpha /\left(1+\left(P / P_{0}\right)^{n}\right)$. The parameter $a$ is the rate at which Hes1 protein is produced from Hes1 mRNA and $b$ and $c$ are the decay rates of Hes1 protein and Hes1 mRNA, respectively. $f(P)$ is the rate of production of new mRNA molecules, with $\alpha$ and $P_{0}$ as constants, to represent the rate of transcript initiation in the absence of Hesl protein and the reference concentration of protein, respectively, and $n$ is the Hill coefficient. $\tau_{m}$ and $\tau_{p}$ represent the transcriptional and translational time delays. The units of the parameters are as follows. $a$ is measured in protein molecules per mRNA molecule per minute; $b$ and $c$ are measured in molecules per minute; $\alpha$ is measured in mRNA molecules per diploid cell per minute; $P_{0}$ is measured in molecules and time delays $\tau_{m}$ and $\tau_{p}$ are measured in minute.

In order to reduce the number of parameters, [7] introduced transformations

$$
m=\frac{M}{\alpha}, \quad p=\frac{P}{\alpha a}, \quad p_{0}=\frac{P_{0}}{\alpha a},
$$

under which system (1) takes the following form:

$$
\begin{gathered}
\frac{d m(t)}{d t}=\frac{1}{1+\left(p\left(t-\tau_{m}\right) / p_{0}\right)^{n}}-c m(t), \\
\frac{d p(t)}{d t}=m\left(t-\tau_{p}\right)-b p(t) .
\end{gathered}
$$

Recently, Zhang et al. [8] investigated the stability and Hopf bifurcation of the equilibrium of system (3). By using the method of multiple time scales, they also obtained the normal form on the center manifold of delay differential equations (3).

The diffusion process comes naturally in biology as pointed out by Murray in [9]. Experimental data also suggests 
that many pathways exhibit oscillation in concentrations of substance involved, both temporally and spatially. For example, a type diffusion process appears in a process of assemblage of particles, which is due to the particles spread out as a result of an irregular individual particle's motion. In these cases, a partial differential equation or system of partial differential equations will be employed to describe the processes. Nowadays, models involving delays and also spatial diffusion are increasingly applied to the study due to more appropriate biological justification. Reference [10] gave plausible biological explanations for the delays appearing in the model (3). In what follows, spatial diffusion will be applied to the model (3) to gain new information about the precise spatiotemporal dynamics of mRNA and proteins. In fact, Sturrock et al. [11] derived a system of partial differential equations to capture the evolution in space and time of the variables in the Hes1 and p53-Mdm2 systems. In this paper, we extend previous mathematical model (3) into its spatial version by considering spatial interactions within the cell explicitly (of course, the clear biological explanation will be given behind the model (4) of promotion); namely, we consider the delayed reaction-diffusion system with the following initial and boundary conditions:

$$
\begin{gathered}
\frac{\partial m(x, t)}{\partial t}=d_{1} \frac{\partial^{2} m(x, t)}{\partial x^{2}}+\frac{1}{1+\left(p\left(x, t-\tau_{m}\right) / p_{0}\right)^{n}} \\
-c m(x, t), \quad t>0, \quad x \in(0, \pi), \\
\frac{\partial p(x, t)}{\partial t}=d_{2} \frac{\partial^{2} p(x, t)}{\partial x^{2}}+m\left(x, t-\tau_{p}\right)-b p(x, t), \\
t>0, \quad x \in(0, \pi), \\
\frac{\partial m(x, t)}{\partial x}=\frac{\partial p(x, t)}{\partial x}=0, \quad x=0, \pi, t \geq 0, \\
m(x, t)=\phi(x, t) \geq 0, \quad p(x, t)=\psi(x, t) \geq 0, \\
\quad(x, t) \in[0, \pi] \times[-\tau, 0],
\end{gathered}
$$

where $d_{1}$ and $d_{2}$ are the diffusion coefficients for Hes1 mRNA and Hesl protein, respectively, with unit such as $\mathrm{cm} / \mathrm{min}$. The initial function $\phi(t, x), \psi(t, x) \in \mathscr{C}:=C\left([-\tau, 0], L^{2}([0, \pi])\right)$. The imposed Neumann boundary condition here implies that mRNA and protein are not exported across the nuclear membrane or the cell membrane.

Obviously, (4) is a system of reaction-diffusion equations modeling the spatiotemporal evolution of the Hesl system. The same reaction kinetics from the ODE model (3) are retained but are now also coupled with diffusion to model explicitly protein and mRNA transport in a cell. That is to say, molecules move from the nucleus to the cytoplasm and from cytoplasm to nucleus across the nuclear membrane. Here, we use a system of PDEs (4) to reflect the reality that the mRNA is transcribed from DNA exclusively in the nucleus and that protein is translated from mRNA exclusively in the cytoplasm. The main advantage of using systems of PDEs (4) to model intracellular reactions is that the PDEs enable spatial effects to be examined explicitly. The main object of this paper is to investigate the effect of the delay and diffusion on the dynamics of system (4). In addition, in order to determine the direction and the stability of Hopf bifurcations, we use the normal form procedure for functional differential equations (FDEs) with diffusion due to Faria [12, 13].

This paper is organized as follows. In Section 2, stability of positive equilibrium and existence of Hopf bifurcation are studied using $\tau$ as a parameter. In Section 3, the effect of diffusion on the Hopf bifurcation will be investigated. Using the normal form technique for partial functional differential equations, the formulas for determining the direction and stability of Hopf bifurcation are presented in Section 4. Finally, in Section 5, we will illustrate the theoretical results by numerical simulations along with some discussion.

\section{Stability of Positive Equilibrium and Existence of Hopf Bifurcation}

It is easy to verify that system (4) has a unique positive equilibrium $E^{*}\left(m^{*}, p^{*}\right)$ determined by

$$
\begin{gathered}
\frac{1}{1+\left(p^{*} / p_{0}\right)^{n}}-c m^{*}=0 \\
m^{*}-b p^{*}=0 .
\end{gathered}
$$

The object here is then to relate the dynamics of (3) and (4) in the neighborhood of $E^{*}$, at the first critical point of the parameter $\tau$. To this end, we let $\bar{m}=m-m^{*}, \bar{p}=p-p^{*}$. With the help of (5), after dropping the bars for simplicity of notation, (4) is transformed into the following system:

$$
\begin{gathered}
\frac{\partial m(x, t)}{\partial t}=d_{1} \frac{\partial^{2} m(x, t)}{\partial x^{2}}+\frac{1}{1+\left(\left(p\left(x, t-\tau_{m}\right)+p *\right) / p_{0}\right)^{n}} \\
-c\left(m(x, t)+m^{*}\right), \\
\frac{\partial p(x, t)}{\partial t}=d_{2} \frac{\partial^{2} p(x, t)}{\partial x^{2}}+m\left(x, t-\tau_{p}\right)-b p(x, t)
\end{gathered}
$$

with the origin as its equilibrium.

Let

$$
\begin{aligned}
f^{(1)}(m, p)= & \frac{1}{1+\left(\left(p\left(x, t-\tau_{m}\right)+p *\right) / p_{0}\right)^{n}} \\
& -c\left(m(x, t)+m^{*}\right), \\
f^{(2)}(m, p) & =m\left(x, t-\tau_{p}\right)-b p(x, t) .
\end{aligned}
$$

Define $f_{i j}^{(1)}(i+j \geq 1)$ and $f_{i j}^{(2)}(i+j \geq 1)$ as follows:

$$
f_{i j}^{(1)}=\frac{\partial^{i+j} f^{(1)}(0,0)}{\partial m^{i} \partial p^{j}}, \quad f_{i j}^{(2)}=\frac{\partial^{i+j} f^{(2)}(0,0)}{\partial m^{i} \partial p^{j}}
$$


where $i$ and $j$ are the nonnegative integers. Then system (6) can be rewritten as

$$
\begin{aligned}
\frac{\partial m(x, t)}{\partial t}= & d_{1} \frac{\partial^{2} m(x, t)}{\partial x^{2}}+a_{11} m(x, t)+a_{12} p\left(x, t-\tau_{m}\right) \\
& +\sum_{i+j \geq 2} \frac{1}{i ! j !} f_{i j}^{(1)} m^{i}(x, t) p^{j}\left(x, t-\tau_{m}\right), \\
\frac{\partial p(x, t)}{\partial t}= & d_{2} \frac{\partial^{2} p(x, t)}{\partial x^{2}}+a_{21} m\left(x, t-\tau_{p}\right)+a_{22} p(x, t) \\
& +\sum_{i+j \geq 2} \frac{1}{i ! j !} f_{i j}^{(2)} m^{i}\left(x, t-\tau_{p}\right) p^{j}(x, t),
\end{aligned}
$$

where

$$
\begin{gathered}
a_{11}=f_{10}^{(1)}=\frac{\partial f^{(1)}}{\partial m}(0,0)=-c \\
a_{12}=f_{01}^{(1)}=\frac{\partial f^{(1)}}{\partial p}(0,0)=-\frac{n c^{2} b^{2}\left(p^{*}\right)^{n+1}}{p_{0}^{n}}, \\
a_{21}=f_{10}^{(2)}=\frac{\partial f^{(2)}}{\partial m}(0,0)=1, \\
a_{22}=f_{01}^{(2)}=\frac{\partial f^{(2)}}{\partial p}(0,0)=-b .
\end{gathered}
$$

For simplification of notation, we use $m(t)$ for $m(\cdot, t)$ and $p(t)$ for $p(\cdot, t)$ and $(m(t), p(t))=(m(\cdot, t), p(\cdot, t))$ is in a suitable Hilbert space $X$

$$
X=\left\{(m, p): m, p \in W^{2,2}(0, \pi), \frac{\partial m}{\partial x}=\frac{\partial p}{\partial x}=0 \text { at } x=0, \pi\right\} .
$$

By setting $U(t)=(m(t), p(t)) \in X$, we further write (9) as an abstract equation in $\mathscr{C}:=C([-\tau, 0], X)$ :

$$
\frac{d U(t)}{d t}=d \Delta U(t)+L\left(U_{t}\right)+F\left(U_{t}\right)
$$

where $d \Delta=\left(d_{1} \Delta, d_{2} \Delta\right)$ and $L: \mathscr{C} \rightarrow R^{2}$, and $F: \mathscr{C} \times R \rightarrow$ $R^{2}$ are given by

$$
\begin{gathered}
L(\varphi)=\left(\begin{array}{c}
a_{11} \varphi_{1}(0)+a_{12} \varphi_{2}\left(-\tau_{m}\right) \\
a_{21} \varphi_{1}\left(-\tau_{p}\right)+a_{22} \varphi_{2}(0)
\end{array}\right), \\
F(\varphi)=\left(\begin{array}{c}
\sum_{i+j \geq 2} \frac{1}{i ! j !} f_{i j}^{(1)} \varphi_{1}^{i}(0) \varphi_{2}^{j}\left(-\tau_{m}\right) \\
\sum_{i+j \geq 2} \frac{1}{i ! j !} f_{i j}^{(2)} \varphi_{1}^{i}\left(-\tau_{p}\right) \varphi_{2}^{j}(0)
\end{array}\right),
\end{gathered}
$$

for $\varphi=\left(\varphi_{1}, \varphi_{2}\right) \in \mathscr{C}$. Obviously, $L$ is a linear operator. The linearization of (12) is

$$
\frac{d U(t)}{d t}=d \Delta U(t)+L\left(U_{t}\right) .
$$

It has a characteristic equation given by

$$
\Delta_{k}(\lambda, \tau)=\lambda^{2}+p_{k} \lambda+r_{k}+K e^{-2 \lambda \tau}=0, \quad k=0,1,2, \ldots,
$$

where $p_{k}=\left(d_{1}+d_{2}\right) k^{2}+(b+c), r_{k}=\left(d_{1} k^{2}+c\right)\left(d_{2} k^{2}+b\right)$, $K=n c^{2} b^{2}\left(p^{*}\right)^{n+1} / p_{0}^{n}$, and $2 \tau=\tau_{m}+\tau_{p}$ is the total time delay. When there is no diffusion effect, namely, $d_{1}=d_{2}=0$, (15) can be written as

$$
\lambda^{2}+(b+c) \lambda+b c+K e^{-2 \lambda \tau}=0,
$$

which is equivalent to (12) of Zhang et al's work in [8], and the related stability and Hopf bifurcation have been investigated.

In what follows, we will analyze the effect of diffusion terms by the distribution of the roots of (15) with $d_{1}>0$, $d_{2}>0$. We first consider the case when the delay is zero. For (15), if $\tau=0$, then we have

$$
\lambda^{2}+p_{k} \lambda+r_{k}+K=0, \quad k=0,1,2 .
$$

Since $p_{k}>0, r_{k}>0$ for any $k \in N$ and $K>0$, it is easy to verify that (17) has a pair of roots with negative real parts. And, for $\tau>0$, we have the following lemma.

Lemma 1. Assume that

$$
\left(d_{1}+c\right)\left(d_{2}+b\right) \geq K
$$

holds. Then all the roots of the characteristic equation (15) have negative real part for $\tau>0$.

Proof. If the conclusion is not true, namely, (15) admits at least one root $\lambda=\mu+i \omega$ with $\mu \geq 0$, then we obtain

$$
(\mu+i \omega)^{2}+p_{k}(\mu+i \omega)+r_{k}+K e^{-2 \tau(\mu+i \omega)}=0 .
$$

Separating the real and imaginary parts yields

$$
\begin{gathered}
\mu^{2}-\omega^{2}+p_{k} \mu+r_{k}+K e^{-\mu \cdot 2 \tau} \cos (\omega \cdot 2 \tau)=0, \\
2 \mu \omega+p_{k} \omega-K e^{-\mu \cdot 2 \tau} \sin (\omega \cdot 2 \tau)=0 .
\end{gathered}
$$

It implies that

$$
\left(\mu^{2}-\omega^{2}+p_{k} \mu+r_{k}\right)^{2}+\left(2 \mu \omega+p_{k} \omega\right)^{2}=K^{2} e^{-2 \mu \cdot 2 \tau}
$$

namely,

$$
\begin{aligned}
\mu^{4}+ & \omega^{4}+p_{k}^{2} \mu^{2}+r_{k}^{2}+2 \mu^{2} \omega^{2}+2 p_{k} \mu^{3} \\
& +2 \mu^{2} \omega^{2}+\left(p_{k}^{2}-2 r_{k}\right) \omega^{2}+2 p_{k} \mu r_{k}=K^{2} e^{-2 \mu \cdot 2 \tau}
\end{aligned}
$$

Since $p_{k}^{2}-2 r_{k}=k^{4}\left(d_{1}^{2}+d_{2}^{2}\right)+2\left(b d_{2}+c d_{1}\right) k^{2}+b^{2}+c^{2}>0$, we can easily verify

$$
r_{k}^{2}<K^{2} e^{-2 \mu \cdot 2 \tau}<K^{2} .
$$

That is, $r_{k}<K$. Notice that $\left(d_{1}+c\right)\left(d_{2}+b\right) \leq r_{k}$, for $k \geq 1$, $k \in N$, gives

$$
\left(d_{1}+c\right)\left(d_{2}+b\right)<K
$$

It is a contradiction. Thus the conclusion follows. 
Notice that when $\tau=0$, all roots of (15) have negative real part and the roots of (15) continuously depend on the parameter $\tau$, and we can summarize our conclusion as follows.

Theorem 2. Assume that $(H)$ holds. Then the equilibrium point $E^{*}$ of system (4) is asymptotically stable for $\tau \geq 0$.

\section{Effects of Diffusion on the Hopf Bifurcation}

Assume that $i \omega(\omega>0)$ is a purely imaginary root of (15); then we have

$$
\begin{gathered}
-\omega^{2}+r_{k}+K \cos (\omega \cdot 2 \tau)=0, \\
p_{k} \omega-K \sin (\omega \cdot 2 \tau)=0,
\end{gathered}
$$

which implies that

$$
\omega^{4}+P_{k} \omega^{2}+R_{k}=0, \quad k=0,1,2, \ldots,
$$

where

$$
\begin{aligned}
P_{k}= & p_{k}^{2}-2 r_{k} \\
= & \left(d_{1}^{2}+d_{2}^{2}\right) k^{4}+2\left(b d_{2}+c d_{1}\right) k^{2}+b^{2}+c^{2}>0, \\
R_{k}=r_{k}^{2}-K^{2}= & \left(r_{k}+K\right)\left(r_{k}-K\right) \\
= & {\left[\left(d_{1} k^{2}+c\right)\left(d_{2} k^{2}+c\right)+K\right] } \\
& \times\left[\left(d_{1} k^{2}+c\right)\left(d_{2} k^{2}+c\right)-K\right] .
\end{aligned}
$$

Equation (25) implies that $R_{k}$ should be negative for some $k \epsilon$ $\mathbf{N}$. It is equivalent to the fact that

$$
\widetilde{R}_{k}=\left(d_{1} k^{2}+c\right)\left(d_{2} k^{2}+c\right)-K
$$

should be less than 0 as it is easy to see that $R_{k}$ has the same sign as that of $\widetilde{R}_{k}$. Rewrite $\widetilde{R}_{k}$ into the following form:

$$
\widetilde{R}_{k}=d_{1} d_{2} k^{4}+\left(d_{1} b+d_{2} c\right) k^{2}+b c-K .
$$

It is obviously a quadratic polynomial in terms of $k^{2}$. Equation (28) implies that there exists a $k_{1} \in \mathrm{N}$ such that $\widetilde{R}_{k_{1}}<0$ if and only if $b c-K<0$. Furthermore, we have

$$
\begin{gathered}
\widetilde{R}_{k}<0 \quad \text { for } 0 \leq k \leq k_{1}, \\
\widetilde{R}_{k}>0 \quad \text { for } k>k_{1}, k \in \mathbb{N} .
\end{gathered}
$$

From (29), we obtain that, for each $k \in\left\{0,1, \ldots, k_{1}\right\},(25)$ has only one positive real root $\omega_{k}$, which is given by

$$
\omega_{k}=\frac{\sqrt{2}}{2} \sqrt{-P_{k}+\sqrt{P_{k}^{2}-4 R_{k}}}
$$

We now can make the following conclusion.
Lemma 3. Assume that $(H)$ is not true. Then (15) has a pair of purely imaginary roots $\pm i \omega_{k}$ for each $k \in\left\{0,1, \ldots, k_{1}\right\}$ and has no purely imaginary roots for $k_{1}<k \in \mathbf{N}$, where $k_{1}$ and $\omega_{k}$ are defined as above. $k_{1}$.

In the rest of this section, we will discuss the case of $k<$ From (24), we have

$$
\sin (\omega \cdot 2 \tau)=\frac{p_{k} \omega}{K}, \quad \cos (\omega \cdot 2 \tau)=\frac{\omega^{2}-r_{k}}{K} .
$$

Then, for $k \in\left\{0,1, \ldots, k_{1}\right\}$, define

$$
\tau_{j}^{k}=\frac{1}{\omega_{k}}\left(\arccos \frac{\omega_{k}^{2}-r_{k}}{K}+2 j \pi\right), \quad j=0,1,2, \ldots
$$

In the following, we will order the sequence of $\tau_{j}^{k}$ depending on the diffusion coefficients $d_{1}$ and $d_{2}$ for $k \in\left\{0,1, \ldots, k_{1}\right\}$.

Lemma 4. If $d_{1}=d_{2}$ and $(\bar{H})$ holds, then

$$
\tau_{0}^{0}=\min \left\{\tau_{j}^{k}\right\}_{k \in\left\{0,1, \ldots, k_{1}\right\}}, \quad j=0,1,2, \ldots
$$

Proof. If $d_{1}=d_{2}$, from (30), we have

$$
\begin{aligned}
& \omega_{k}^{2}=\frac{1}{2}[-(b+c)^{2}-2(b+c) d k^{2}-2 d^{2} k^{4}+2 b c \\
&\left.+\sqrt{(b-c)^{2}\left[2 d k^{2}+(b+c)\right]^{2}+4 K^{2}}\right], \\
& \omega_{k}^{2}-r_{k}=\frac{1}{2}[-(b+c)^{2}-4(b+c) d k^{2}-4 d^{2} k^{4} \\
&\left.+\sqrt{(b-c)^{2}\left[2 d k^{2}+(b+c)\right]^{2}+4 K^{2}}\right] .
\end{aligned}
$$

Let $x=\sqrt{(b-c)^{2}\left[2 d k^{2}+(b+c)\right]^{2}+4 K^{2}}$; it is easy to verify

$$
x>(b-c)^{2},
$$

$$
\begin{gathered}
\omega_{k}^{2}=\frac{1}{2}\left[x-\frac{x^{2}-4 K^{2}}{2(b-c)^{2}}+\frac{(b-c)^{2}}{2}\right], \\
\omega_{k}^{2}-r_{k}=\frac{1}{2}\left[x-\frac{x^{2}-4 K^{2}}{2(b-c)^{2}}\right] .
\end{gathered}
$$

Thus, according to (32), we obtain

$\tau_{j}^{k}=\tau(x)$

$$
=\frac{\arccos \left[\left(x-\left(\left(x^{2}-4 K^{2}\right) /(b-c)^{2}\right) / 2 K\right)+2 j \pi\right]}{(\sqrt{2} / 2)\left[x-\left(\left(x^{2}-4 K^{2}\right) / 2(b-c)^{2}\right)+\left((b-c)^{2} / 2\right)\right]^{1 / 2}} .
$$

In addition,

$$
\frac{d\left(x-\left(\left(x^{2}-4 K^{2}\right) / 2(b-c)^{2}\right)\right)}{d x}=1-\frac{x}{(b-c)^{2}} .
$$


From (35), we know that $x-\left(\left(x^{2}-4 K^{2}\right) / 2(b-c)^{2}\right)$ is decreasing with respect to $x$. Then we obtain $\tau_{j}^{k+1}>\tau_{j}^{k}$ if $d_{1}=d_{2}$. Clearly, $\tau_{j+1}^{k}>\tau_{j}^{k}$, so we have

$$
\tau_{0}^{0}=\min \left\{\tau_{j}^{k}\right\}_{k \in\{0,1,2, \ldots\}}, \quad j=0,1,2, \ldots
$$

According to Lemma 4 and the continuous dependence of $\tau_{j}^{k}$ on $d_{1}$ and $d_{2}$, we summarize the following lemma.

Lemma 5. For any $d>0$, there exists an $\epsilon(d)>0$ such that, for any $d_{1}, d_{2} \in(d-\epsilon, d+\epsilon)$ satisfying $(\bar{H}), \tau_{0}^{0}=$ $\min \left\{\tau_{j}^{k}\right\}_{k \in\left\{0,1, \ldots, k_{1}\right\}}, \quad j=0,1,2, \ldots$..

Let $\lambda_{k}(\tau)=\mu_{k}(\tau)+i \omega_{k}(\tau)$ be the roots of (15) near $2 \tau=\tau_{j}^{k}$ satisfying $\mu_{k}\left(\tau_{j}^{k}\right)=0, \omega_{k}\left(\tau_{j}^{k}\right)=\omega_{k}$. By using the method in $[14,15]$, we can prove the following transversality condition.

Lemma 6. If $d_{1}$ and $d_{2}$ satisfy the condition in Lemma 5 , then, for $k \in\left\{0,1, \ldots, k_{1}\right\}$ and $j \in \mathbb{N}_{0}, d \operatorname{Re}(\lambda) /\left.d \tau\right|_{2 \tau=\tau_{j}^{k}}>0$.

Proof. Differentiating equation (15) with respect to $\tau$, we obtain

$$
\left(\frac{d \lambda}{d \tau}\right)^{-1}=\frac{\left(2 \lambda+p_{k}\right) e^{2 \lambda \tau}}{2 \lambda K}-\frac{\tau}{\lambda}
$$

From (24), we have

$$
\begin{aligned}
\operatorname{Re}\left(\left.\frac{d \lambda}{d \tau}\right|_{2 \tau=\tau_{j}^{k}}\right)^{-1} & =\frac{2 \omega_{k} \cos \left(\omega_{k} \tau_{j}^{k}\right)+p_{k} \sin \left(\omega_{k} \tau_{j}^{k}\right)}{2 k \omega_{k}} \\
& =\frac{2 \omega_{k} \cdot\left(\left(\omega_{k}^{2}-r_{k}\right) / K\right)+p_{k} \cdot\left(p_{k} \omega_{k} / K\right)}{2 k \omega_{k}} \\
& =\frac{2 \omega_{k}^{2}+\left(p_{k}^{2}-2 r_{k}\right)}{2 K^{2}}>0
\end{aligned}
$$

since $p_{k}^{2}-2 r_{k}>0$.

Combining the above analysis and the qualitative theory of partial functional differential equations in [16], we have the following results on the stability of equilibrium $E^{*}$ of system (4) and existence of Hopf bifurcation near $E^{*}$.

Theorem 7. Assume that $d_{1}, d_{2}$ satisfy the condition in Lemma 5. Then, for system (4),

(i) the positive equilibrium $E^{*}$ is asymptotically stable for $\tau \in\left[0, \tau_{0}^{0}\right)$ and unstable for $\tau \in\left(\tau_{0}^{0},+\infty\right)$;

(ii) it undergoes Hopf bifurcations near the positive equilibrium $E^{*}$ at $2 \tau=\tau_{j}^{k}$ for $k \in\left\{0,1, \ldots, k_{1}\right\}$ and $j \in \mathbb{N}_{0}$.

Remark 8. If $d_{1}=d_{2}=0$, Theorem 2 and Theorem 7 are the conclusion of Theorem 1 in [8]. That is, assuming either condition $(H)$ or $(\bar{H})$, Theorem 2 and Theorem 7 show that the local stability of $E^{*}$ for $0 \leq \tau<\tau_{0}^{0}$ is the same for system (3) and system (4). Here we can know the effect of the diffusion coefficients $d_{1}$ and $d_{2}$, or, in order to more clearly understand the effect of diffusion, we take $b=c=0.03$, $p_{0}=0.4, n=2$, and then we get $p^{*}=5.6134, K \approx 17.91 \times 10^{-4}$ by calculation. In the absence of diffusion, $b \times c=9 \times 10^{-4}<K$, it is known that the equilibrium $E^{*}$ of system (3) is stable when $\tau<\tau_{0}^{0}$ according to Theorem 1 in [8]. In the presence of diffusion, for example, taking $d_{1}=0.008, d_{2}=0.02$, which implies $\left(d_{1}+c\right)\left(d_{2}+b\right)=19 \times 10^{-4}>K$, we know that the equilibrium $E^{*}$ of system (4) is stable for $\tau \geq 0$ by Theorem 2 .

\section{The Direction and Stability of Hopf Bifurcation}

In this section, we assume the hypotheses of Theorem 7 hold and $\tau_{m}=\tau_{p}=\tau$. For the case of $\tau_{m} \neq \tau_{p}$, which is not our concern in this paper, the calculation of the normal form should follow the method developed in [17]. By using the normal form method in [12] for partial differential equations with time delay, we will investigate the stability of these Hopf bifurcations. For standard notations and classical results on partial functional differential equations, please refer to [12, $13,17]$. More details on techniques for computing the normal form can also be found in recent work [18].

Now, normalizing by the time-scaling $t \rightarrow t / \tau$, then (12)(13) can be rewritten as

$$
\frac{d U(t)}{d t}=\tau d \Delta U(t)+L(\tau)\left(U_{t}\right)+f\left(U_{t}, \tau\right)
$$

where

$$
\begin{gathered}
L(\tau)(\varphi)=\tau\left(\begin{array}{c}
a_{11} \varphi_{1}(0)+a_{12} \varphi_{2}(-1) \\
a_{21} \varphi_{1}(-1)+a_{22} \varphi_{2}(0)
\end{array}\right), \\
f(\varphi, \tau)=\tau\left(\begin{array}{c}
\sum_{i+j \geq 2} \frac{1}{i ! j !} f_{i j}^{(1)} \varphi_{1}^{i}(0) \varphi_{2}^{j}(-1) \\
\sum_{i+j \geq 2} \frac{1}{i ! j !} f_{i j}^{(2)} \varphi_{1}^{i}(-1) \varphi_{2}^{j}(0)
\end{array}\right) .
\end{gathered}
$$

In the following, we denote any one of these critical values by $\tau_{*}$ at which the characteristic equation (15) has a pair of simply purely imaginary roots $\pm i \omega_{*}$. Let $\tau=\tau_{*}+\alpha, \alpha \in$ $\mathbb{R}$, and consider only the case $\Lambda_{0}=\left\{-i \tau_{*} \omega_{*}, i \tau_{*} \omega_{*}\right\}$ is the set of eigenvalues on the imaginary axis of the infinitesimal generator associated with the flow of

$$
\frac{d U(t)}{d t}=\tau^{*} d \Delta U(t)+L\left(\tau_{*}\right)\left(U_{t}\right) .
$$

Equation (42) is now written as

$$
\frac{d U(t)}{d t}=\tau d \Delta U(t)+L(\tau)\left(U_{t}\right)+F\left(U_{t}, \alpha\right)
$$

where $F(\varphi, \alpha)=\alpha d \Delta \varphi(0)+L(\alpha)(\varphi)+f\left(\varphi, \tau_{*}+\alpha\right)$, for $\varphi \in \mathscr{C}$. The eigenvalues of $\tau_{*} d \Delta$ on $X$ are $\mu_{k}^{i}=-d_{i} \tau_{*} k^{2}, i=1,2$, 
$k \in \mathbb{N}_{0}$, with corresponding normalized eigenfunctions $\beta_{k}^{i}$, where

$$
\begin{gathered}
\beta_{k}^{1}(x)=\left(\begin{array}{c}
\gamma_{k}(x) \\
0
\end{array}\right), \quad \beta_{k}^{2}(x)=\left(\begin{array}{c}
0 \\
\gamma_{k}(x)
\end{array}\right), \\
\gamma_{k}(x)=\frac{\cos (k x)}{\|\cos (k x)\|_{2,2}}, \\
k \in \mathbb{N}_{0} .
\end{gathered}
$$

Let $\mathscr{B}_{k}=\operatorname{span}\left\{\left\langle v(\cdot), \beta_{k}^{i}\right\rangle \beta_{k}^{i} \mid v \in \mathscr{C}, i=1,2\right\}$. Assume that $z_{t}(\theta) \in C=C\left([-1,0], \mathbb{R}^{2}\right)$ and

$$
z_{t}^{T}(\theta)\left(\begin{array}{c}
\beta_{k}^{1} \\
\beta_{k}^{2}
\end{array}\right) \in \mathscr{B}_{k} .
$$

Then linear PFDE (44) restricted to $\mathscr{B}_{k}$ is equivalent to the FDE on $C\left([-1,0], \mathbb{R}^{2}\right)$

$$
\dot{z}(t)=\left(\begin{array}{cc}
\mu_{k}^{1} & 0 \\
0 & \mu_{k}^{2}
\end{array}\right) z(t)+L\left(\tau_{*}\right)\left(z_{t}\right)
$$

with the characteristic equation given by (15).

Suppose that there exists a $k \in \mathbb{N}_{0}$ such that when $\tau=\tau_{*}$, (15) for fixed $k$ has a pair of purely imaginary roots $\pm i \omega_{*}$ and all other roots of (15) have negative real parts. Define $\eta(\theta) \in$ $B V([-1,0] ; R)$ such that

$$
\mu_{k} \psi(0)+L\left(\tau_{*}\right) \psi=\int_{-1}^{0} d \eta(\theta) \psi(\theta), \quad \psi \in C,
$$

and the adjoint bilinear form on $C^{*} \times C, C^{*}=C\left([0,1], \mathbb{R}^{2 *}\right)$,

$$
\begin{array}{r}
(\psi(s), \phi(\theta))=\psi(0) \phi(0)-\int_{-1}^{0} \int_{0}^{\theta} \psi(\xi-\theta) d \eta(\theta) \phi(\xi) d \xi \\
\text { for } \psi \in C^{*}, \phi \in C .
\end{array}
$$

For (48) with fixed $k$, choose a basis $\Psi_{k}$ for the adjoint space $P^{*}$ and a basis $\Phi_{k}$ for its the eigenspace $P$ as follows:

$$
\begin{gathered}
\Phi_{k}=\left(p e^{i \omega_{*} \tau_{*} \theta}, \bar{p} e^{-i \omega_{*} \tau_{*} \theta}\right), \\
\Psi_{k}=\operatorname{col}\left(q^{T} e^{-i \omega_{*} \tau_{*} s}, \bar{q}^{T} e^{i \omega_{*} \tau_{*} s}\right)
\end{gathered}
$$

such that $\left(\Phi_{k}, \Phi_{k}\right)=I_{2}$, where $I_{2}$ is a $2 \times 2$ identity matrix. Then we can easily have

$$
\begin{gathered}
p=\left(\begin{array}{l}
p_{1} \\
p_{2}
\end{array}\right)=\left(\frac{i \omega_{*}+d_{1} k^{2}-a_{11}}{a_{12}} e^{i \omega_{*} \tau_{*}}\right), \\
q=\left(\begin{array}{l}
q_{1} \\
q_{2}
\end{array}\right)=q_{1}\left(\frac{i \omega_{*}+d_{1} k^{2}-a_{11}}{a_{21}} e^{i \omega_{*} \tau_{*}}\right),
\end{gathered}
$$

with

$$
\begin{gathered}
q_{1}=\left(1+2 \tau_{*}\left(i \omega_{*}+d_{1} k^{2}-a_{11}\right)\right. \\
\left.+\frac{\left(i \omega_{*}+d_{1} k^{2}-a_{11}\right)^{2} e^{i \omega_{*} \tau_{*}}}{a_{12} a_{21}}\right)^{-1} .
\end{gathered}
$$

Following the standard procedure in [12], especially [18], using the decomposition $\varphi(t)=\left(\Phi_{k} z\right)^{T}\left(\begin{array}{c}\beta_{k}^{1} \\ \beta_{k}^{2}\end{array}\right)+y, z(t)=$ $\left(\Psi_{k},\left(\begin{array}{c}\left\langle\varphi(\cdot), \beta_{k}^{1}\right\rangle \\ \left\langle\varphi(\cdot), \beta_{k}^{2}\right\rangle\end{array}\right)\right) \in \mathbb{R}^{2}, y(t) \in \mathscr{C}_{0}^{1} \cap \operatorname{Ker} \pi=\mathscr{C}_{0}^{1} \cap \mathbb{Q}:=\mathbb{Q}^{1}$, we decompose (45) as

$$
\begin{gathered}
\dot{z}=B z+\Psi_{k}(0)\left(\begin{array}{c}
\left\langle F\left(\left(\Phi_{k} z\right)^{T}\left(\begin{array}{c}
\beta_{k}^{1} \\
\beta_{k}^{2}
\end{array}\right)+y, \alpha\right), \beta_{k}^{1}\right\rangle \\
\left\langle F\left(\left(\Phi_{k} z\right)^{T}\left(\begin{array}{l}
\beta_{k}^{1} \\
\beta_{k}^{2}
\end{array}\right)+y, \alpha\right), \beta_{k}^{2}\right\rangle
\end{array}\right), \\
\frac{d}{d t} y=A_{Q^{1}} y+(I-\pi) X_{0} F\left(\left(\Phi_{k} z\right)^{T}\left(\begin{array}{c}
\beta_{k}^{1} \\
\beta_{k}^{2}
\end{array}\right)+y, \alpha\right),
\end{gathered}
$$

where here and throughout this section we refer to $[12,18]$ for results and explanations of several notations involved.

Consider the formal Taylor expansion

$$
F(v, \alpha)=\sum_{j \geq 2} \frac{1}{j !} F_{j}(v, \alpha),
$$

where $F_{j}$ is the $j$ th Fréchet derivative of $F$. Then (55) can be written as

$$
\begin{gathered}
\dot{z}=B z+\sum_{j \geq 2} \frac{1}{j !} f_{j}^{1}(z, y, \alpha), \\
\frac{d}{d t} y=A_{Q^{1}} y+\sum_{j \geq 2} \frac{1}{j !} f_{j}^{2}(z, y, \alpha),
\end{gathered}
$$

where $f_{j}^{1}(z, y, \alpha)$ and $f_{j}^{2}(z, y, \alpha)$ are given by (4.8) in [18]. Then (45) has a normal form on the center manifold of the origin at $\alpha=0$, written as

$$
\dot{z}=B z+\frac{1}{2} g_{2}^{1}(z, 0, \alpha)+\frac{1}{3 !} g_{3}^{1}(z, 0,0)+O\left(\alpha^{2}|z|+\alpha|z|^{2}\right),
$$

where $B=\operatorname{diag}\left\{i \omega_{*} \tau_{*},-i \omega_{*} \tau_{*}\right\}$ and $g_{j}^{1}, j=2,3$, are given by (4.9) in [18]. The normal form procedure will show that these terms have the form

$$
\frac{1}{2} g_{2}^{1}(z, 0, \alpha)=\left(\frac{A_{k 1} z_{1} \alpha}{\bar{A}_{k 1} z_{2} \alpha}\right),
$$


where

$$
A_{k 1}=-k^{2}\left(d_{1} q_{1} p_{1}+d_{2} q_{2} p_{2}\right)+i \omega_{*} q^{T} p ;
$$

$\bar{A}_{k 1}$ is the conjugate of $A_{k 1}$. Consider the following:

$$
\frac{1}{3 !} g_{3}^{1}(z, 0,0)=\left(\begin{array}{c}
A_{k 2} z_{1}^{2} z_{2} \\
\bar{A}_{k 2} z_{1} z_{2}^{2}
\end{array}\right),
$$

where

$$
\begin{aligned}
A_{k 2}= & \frac{i}{2 \omega_{*} \tau_{*}}\left(a_{k 20} a_{k 11}-2\left|a_{k 11}\right|^{2}-\frac{1}{3}\left|a_{k 02}\right|^{2}\right) \\
& +\frac{1}{2}\left(a_{k 21}+\frac{1}{2} b_{k 21}\right)
\end{aligned}
$$

with

$$
\begin{aligned}
& a_{k 20}=\tau_{*} \int_{0}^{\pi} \gamma_{k}^{3}(x) d x\left(b_{1} q_{1}\right) \\
& =\left\{\begin{array}{ll}
\frac{\tau_{*}}{\sqrt{\pi}}\left(b_{1} q_{1}\right), & k=0, \\
0, & k \neq 0,
\end{array} \quad b_{1}=f_{02}^{(1)} p_{2}^{2} e^{-2 i \omega_{*} \tau_{*}},\right. \\
& a_{k 11}=\tau_{*} \int_{0}^{\pi} \gamma_{k}^{3}(x) d x\left(b_{3} q_{1}\right) \\
& =\left\{\begin{array}{ll}
\frac{\tau_{*}}{\sqrt{\pi}}\left(b_{3} q_{1}\right), & k=0, \\
0, & k \neq 0,
\end{array} \quad b_{3}=f_{02}^{(1)}\left|p_{2}\right|^{2},\right. \\
& a_{k 02}=\tau_{*} \int_{0}^{\pi} \gamma_{k}^{3}(x) d x\left(\bar{b}_{1} q_{1}\right) \\
& = \begin{cases}\frac{\tau_{*}}{\sqrt{\pi}}\left(\bar{b}_{1} q_{1}\right), & k=0, \\
0, & k \neq 0,\end{cases} \\
& a_{k 21}=\tau_{*} \int_{0}^{\pi} \gamma_{k}^{4}(x) d x\left(q_{1} f_{03}^{(1)} p_{2}\left|p_{2}\right|^{2} e^{-i \omega_{*} \tau_{*}}\right) \\
& = \begin{cases}\frac{\tau_{*}}{\pi}\left(q_{1} f_{03}^{(1)} p_{2}\left|p_{2}\right|^{2} e^{-i \omega_{*} \tau_{*}}\right), & k=0, \\
\frac{3 \tau_{*}}{2 \pi}\left(q_{1} f_{03}^{(1)} p_{2}\left|p_{2}\right|^{2} e^{-i \omega_{*} \tau_{*}}\right), & k \neq 0,\end{cases} \\
& b_{k 21}= \begin{cases}M_{0}, & k=0, \\
M_{0}+\frac{\sqrt{2}}{2} M_{2 k}, & k \neq 0,\end{cases}
\end{aligned}
$$

where, for $j=0,2 k, M_{j}=\left(2 \tau_{*} / \sqrt{\pi}\right) q_{1}\left(f_{02}^{(1)} P_{2} e^{i \omega_{*} \tau_{*}} h_{j 11}^{(2)}(-1)+\right.$ $\left.f_{02}^{(1)} \bar{P}_{2} e^{-i \omega_{*} \tau_{*}} h_{j 11}^{(2)}(-1)\right)$, while $h_{k 20}(\theta)=\bar{h}_{k 02}(\theta)$ and $h_{k 20}(\theta)$, $h_{k 11}(\theta)$ are determined by the following equations:

$$
\begin{gathered}
\dot{h}_{k 20}(\theta)-2 i \tau_{*} \omega_{*} h_{k 20}(\theta)=\Phi_{k}\left(\begin{array}{c}
a_{k 20} \\
\bar{a}_{k 02}
\end{array}\right), \\
\dot{h}_{k 20}(0)-L\left(\tau_{*}\right)\left(h_{k 20}\right)=\tau_{*} c_{k j}\left(\begin{array}{c}
b_{1} \\
0
\end{array}\right), \\
\dot{h}_{k 11}(\theta)=\Phi_{k}\left(\begin{array}{l}
2 a_{k 11} \\
2 \bar{a}_{k 11}
\end{array}\right), \\
\dot{h}_{k 11}(0)-L\left(\tau_{*}\right)\left(h_{k 11}\right)=\tau_{*} c_{k j}\left(\begin{array}{c}
b_{3} \\
0
\end{array}\right),
\end{gathered}
$$

where

$$
c_{k j}=\int_{0}^{\pi} \gamma_{k}^{2}(x) \gamma_{j}(x) d x= \begin{cases}\frac{1}{\sqrt{\pi}}, & j=k=0, \\ \frac{1}{\sqrt{\pi}}, & j=0, k \neq 0, \\ \frac{1}{\sqrt{2 \pi}}, & j=2 k \neq 0, \\ 0 . & \text { otherwise. }\end{cases}
$$

So the normal form (45) on the center manifold has the form

$$
\dot{z}=B z+\left(\begin{array}{l}
A_{k 1} z_{1} \mu \\
\bar{A}_{k 1} z_{2} \mu
\end{array}\right)+\left(\begin{array}{l}
A_{k 2} z_{1}^{2} z_{2} \\
\bar{A}_{k 2} z_{1} z_{2}^{2}
\end{array}\right)+O\left(|z| \alpha^{2}+\left|z^{4}\right|\right) .
$$

Next we will derive the normal form in the real coordinates. To this end, let $z_{1}=w_{1}-i w_{2}, z_{2}=w_{1}+i w_{2}$, and then the polar coordinates $w_{1}=\rho \cos \xi, w_{2}=\rho \sin \xi$. We finally reach

$$
\begin{gathered}
\dot{\rho}=\iota_{k 1} \alpha \rho+\iota_{k 2} \rho^{3}+O\left(\alpha^{2} \rho+|(\rho, \alpha)|^{4}\right), \\
\dot{\xi}=-\omega_{*} \tau_{*}+O(|(\rho, \alpha)|) ;
\end{gathered}
$$

here $\iota_{k 1}=\operatorname{Re} A_{k 1}, \iota_{k 2}=\operatorname{Re} A_{k 2}$. Then, from [19], we know that the number $\iota_{k 2}$ tells the bifurcation direction and the stability of bifurcating periodic solution.

(i) When $t_{k 2}<0$, it is a supercritical bifurcation and the bifurcating periodic solution is stable.

(ii) When $\imath_{k 2}>0$, it is a subcritical bifurcation and the bifurcating periodic solution is unstable.

\section{Numerical Simulation and Discussion}

In this section, we present some numerical simulations to system (4). These simulations are used to support our theoretical results. Similar to $[6,7,20]$, the values of parameters are taken from the published experimental and theoretical results in our simulations where, $n \in[2,10], P_{0} \in[40,100]$, and $\mu \in[0.01,1] . \alpha=33$ and $a=4.5$ are taken as in [10] for original model (1). 

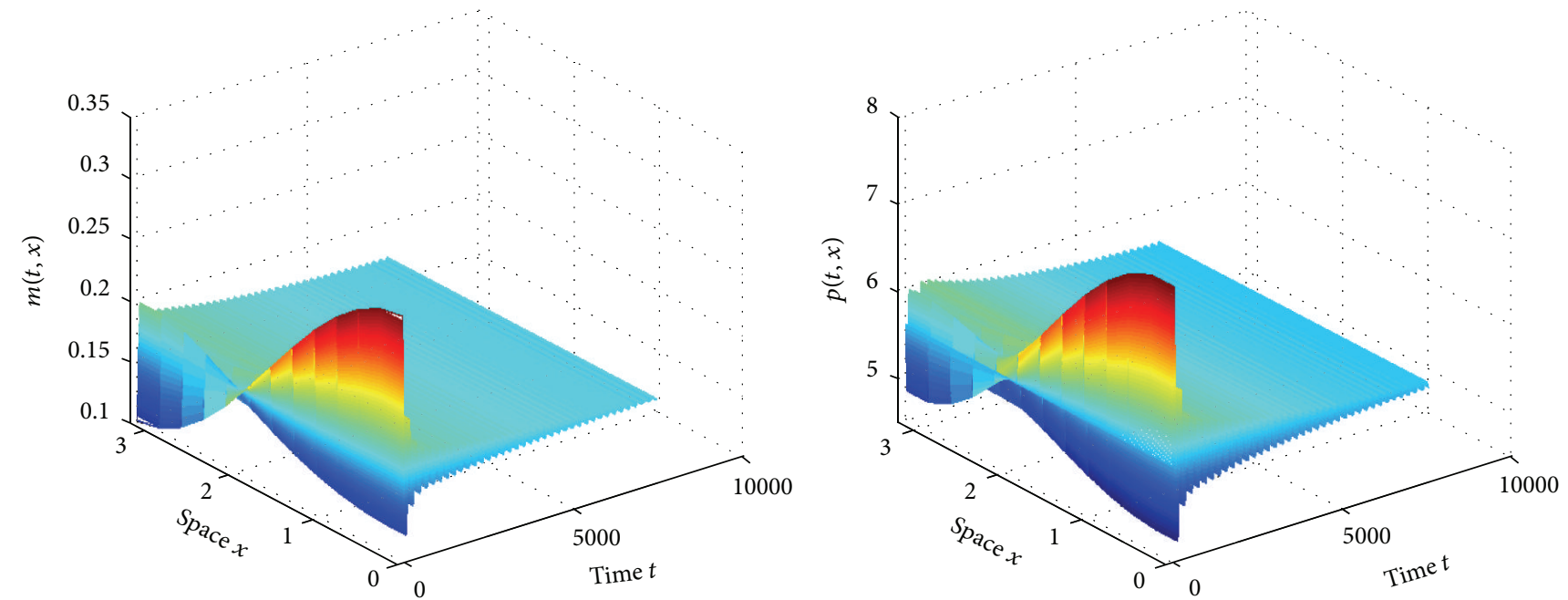

FIGURE 1: Numerical simulations of system (4) with $d_{1}=0.002, d_{2}=0.02, b=c=0.03, n=2, p_{0}=0.4$, and $\tau=25<\tau_{0}^{0}$. The initial values are $m_{0}(x)=0.2+0.1 \cos x ; p_{0}(x)=5.6-0.01 \cos x$. The positive equilibrium $E^{*}(0.1684,5.6134)$ of system $(4)$ is asymptotically stable for $\in\left[0, \tau_{0}^{0}\right)$.
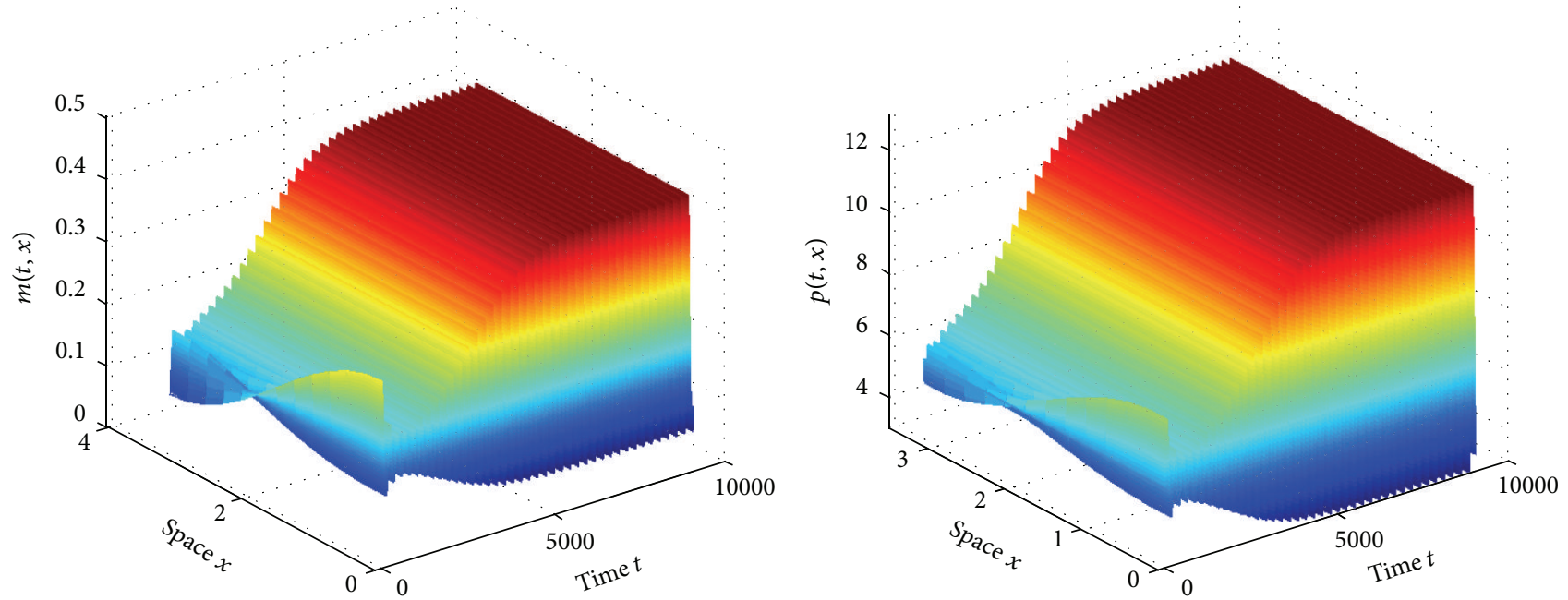

FIgURE 2: Numerical simulations system (4) with $d_{1}=0.002, d_{2}=0.02, b=c=0.03, n=2, p_{0}=0.4$, and $\tau=30>\tau_{0}^{0}$. The initial values are $m_{0}(x)=0.2+0.1 \cos x ; p_{0}(x)=5.6-0.01 \cos x$. The positive equilibrium $E^{*}(0.1684,5.6134)$ of system $(4)$ becomes unstable and there exist stable spatially homogeneous periodic solutions.

Taking $d_{1}=0.002, d_{2}=0.02, n=2, b=c=\mu=0.03$, and $p_{0}=0.4\left(P_{0}=59.4\right)$, then the positive equilibrium $E^{*}\left(m^{*}, p^{*}\right)=(0.1684,5.6134)$. From (30) and (32), we obtain the critical value for time delay, $\tau_{0}^{0} \doteq 26.3983$. In this case, the parameters satisfy $(\bar{H})$. By Theorem 7 , the positive equilibrium $E^{*}(0.1684,5.6134)$ is asymptotically stable for $\tau=25<\tau_{0}^{0}$. Figure 1 is the numerical simulation of system (4) for $\tau=25$.

When the delay increasingly crosses through the critical value $\tau_{0}^{0} \doteq 26.3983$, the positive equilibrium $E^{*}$ loses its stability and the Hopf bifurcation occurs. Taking $\tau=30>\tau_{0}^{0}$,
Figure 2 is the numerical simulation results of system (4). It is consistent with the theoretical results.

In Figure 2, we fix $x=1.5708$ and the other parameter values are the same as Figure 2. Then we get Figure 3 which shows that the oscillation will sustain when the time delay $\tau=30$ is much greater than the critical value $\tau_{0}^{0} \dot{=}$ 26.3983 .

Remark 9. Comparing Figure 3 with Figure 3 in [8], we know that the oscillation is still sustained when $\tau$ is much greater than its critical value $\tau_{0}^{0}$ in the presence of diffusion. 

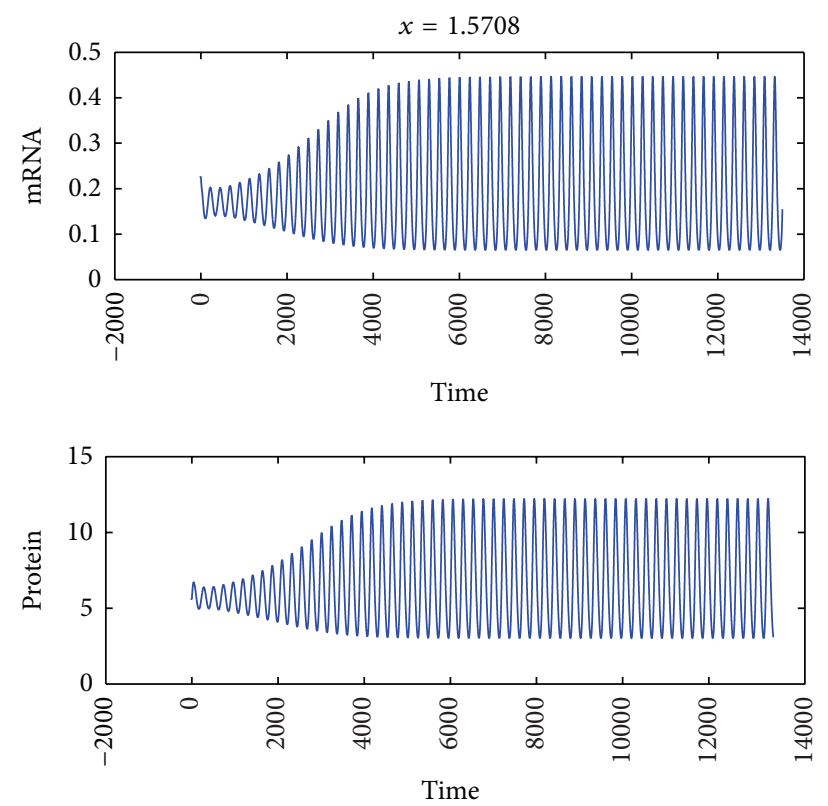

FIGURE 3: Sustained oscillation when $\tau=30$ is much larger than its critical value $\tau_{0}^{0} \doteq 26.3983$ with $d_{1}=0.002, d_{2}=0.02, b=c=0.03$, $n=2, p_{0}=0.4$ and the initial values are $m_{0}(x)=0.2+0.1 \cos x$; and $p_{0}(x)=5.6-0.01 \cos x$.

\section{Conflict of Interests}

The authors declare that there is no conflict of interests regarding the publication of this paper.

\section{Acknowledgments}

The research is supported by the National Natural Science Foundation of China (nos. 11101076 and 11371087), the Shanghai Committee of Science and Technology (nos. 11ZR1400200 and 12ZR1400100), the Fundamental Research Funds for the Central Universities (2232011D-35), and China Scholarship Council.

\section{References}

[1] B. C. Goodwin, "Oscillatory behavior in enzymatic control processes," Advances in Enzyme Regulation, vol. 3, pp. 425-428, 1965.

[2] S. Busenberg and J. Mahaffy, "Interaction of spatial diffusion and delays in models of genetic control by repression," Journal of Mathematical Biology, vol. 22, no. 3, pp. 313-333, 1985.

[3] J. M. Mahaffy, "Genetic control models with diffusion and delays," Mathematical Biosciences, vol. 90, no. 1-2, pp. 519-533, 1988.

[4] J. M. Mahaffy and C. V. Pao, "Models of genetic control by repression with time delays and spatial effects," Journal of Mathematical Biology, vol. 20, no. 1, pp. 39-57, 1984.

[5] M. Sturrock, A. J. Terry, D. P. Xirodimas, A. M. Thompson, and M. A. J. Chaplain, "Spatio-temporal modelling of the Hes1 and NF- $\kappa$ B driven by transcriptional time delays," Current Biology, vol. 13, pp. 1409-1413, 2003.
[6] N. A. M. Monk, "Oscillatory expression of Hes1, p53, and NF$\kappa \mathrm{B}$ driven by transcriptional time delays," Current Biology, vol. 13, no. 16, pp. 1409-1413, 2003.

[7] A. Verdugo and R. Rand, "Hopf bifurcation in a DDE model of gene expression," Communications in Nonlinear Science and Numerical Simulation, vol. 13, no. 2, pp. 235-242, 2008.

[8] T. H. Zhang, Y. Song, and H. Zang, "The stability and Hopf bifurcation analysis of a gene expression model," Journal of Mathematical Analysis and Applications, vol. 395, no. 1, pp. 103113, 2012.

[9] J. D. Murray, Mathematical Biology-I. An Introduction, Springer, Berlin, Germany, 3rd edition, 2002.

[10] J. Lewis, "Autoinhibition with transcriptional delay: a simple mechanism for the zebrafish somitogenesis oscillator," Current Biology, vol. 13, no. 16, pp. 1398-1408, 2003.

[11] M. Sturrock, A. J. Terry, D. P. Xirodimas, A. M. Thompson, and M. A. J. Chaplain, "Spatio-temporal modelling of the Hes1 and p53-Mdm2 intracellular signalling pathways," Journal of Theoretical Biology, vol. 273, pp. 15-31, 2011.

[12] T. Faria, "Normal forms and Hopf bifurcation for partial differential equations with delays," Transactions of the American Mathematical Society, vol. 352, no. 5, pp. 2217-2238, 2000.

[13] T. Faria, "Bifurcation aspects for some delayed population models with diffusion," in Differential Equations with Applications to Biology, vol. 21 of Fields Institute Communications, pp. 143-158, 1999.

[14] E. Beretta and Y. Kuang, "Geometric stability switch criteria in delay differential systems with delay dependent parameters," SIAM Journal on Mathematical Analysis, vol. 33, no. 5, pp. 11441165, 2002.

[15] Y. L. Song and J. J. Wei, "Local Hopf bifurcation and global periodic solutions in a delayed predator-prey system," Journal of Mathematical Analysis and Applications, vol. 301, no. 1, pp. 121, 2005.

[16] J. Wu, Theory and Applications of Partial Functional-Differential Equations, Springer, New York, NY, USA, 1996.

[17] T. Faria, "Stability and bifurcation for a delayed predator-prey model and the effect of diffusion," Journal of Mathematical Analysis and Applications, vol. 254, no. 2, pp. 433-463, 2001.

[18] Y. L. Song, Y. H. Peng, and X. F. Zou, "Persistence, stability and Hopf bifurcation in a diffusive ratio-dependent predatorprey model with delay," International Journal of Bifurcation and Chaos. In press.

[19] S. Wiggins, Introduction to Applied Nonlinear Dynamical Systems and Chaos, Springer, New York, NY, USA, 2nd edition, 2003.

[20] M. H. Jensen, K. Sneppen, and G. Tiana, "Sustained oscillations and time delays in gene expression of protein Hesl," FEBS Letters, vol. 541, no. 1-3, pp. 176-177, 2003. 


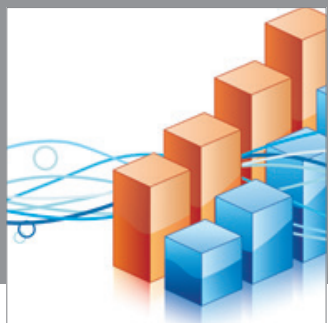

Advances in

Operations Research

mansans

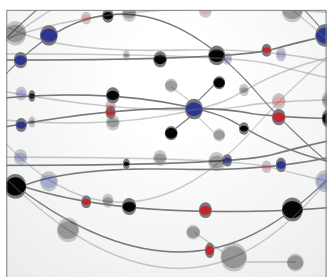

The Scientific World Journal
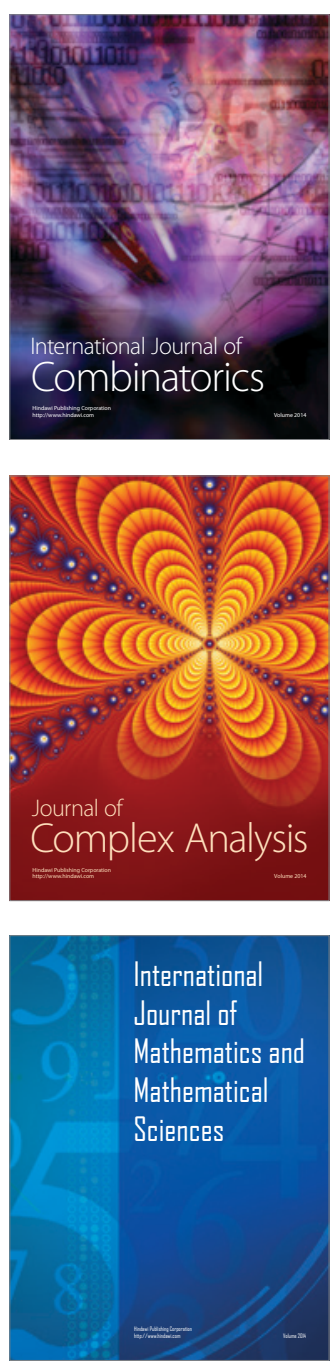
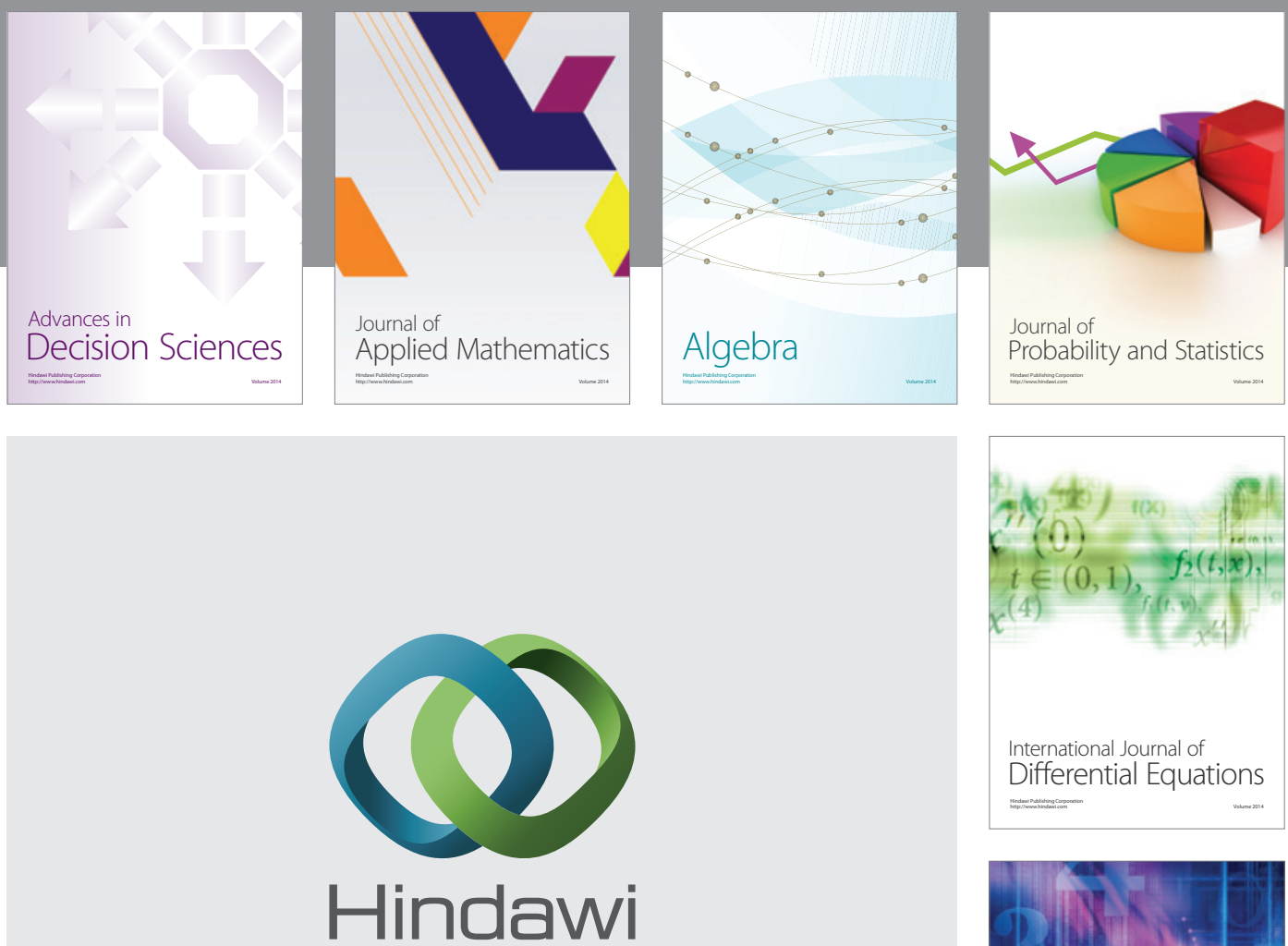

Submit your manuscripts at http://www.hindawi.com
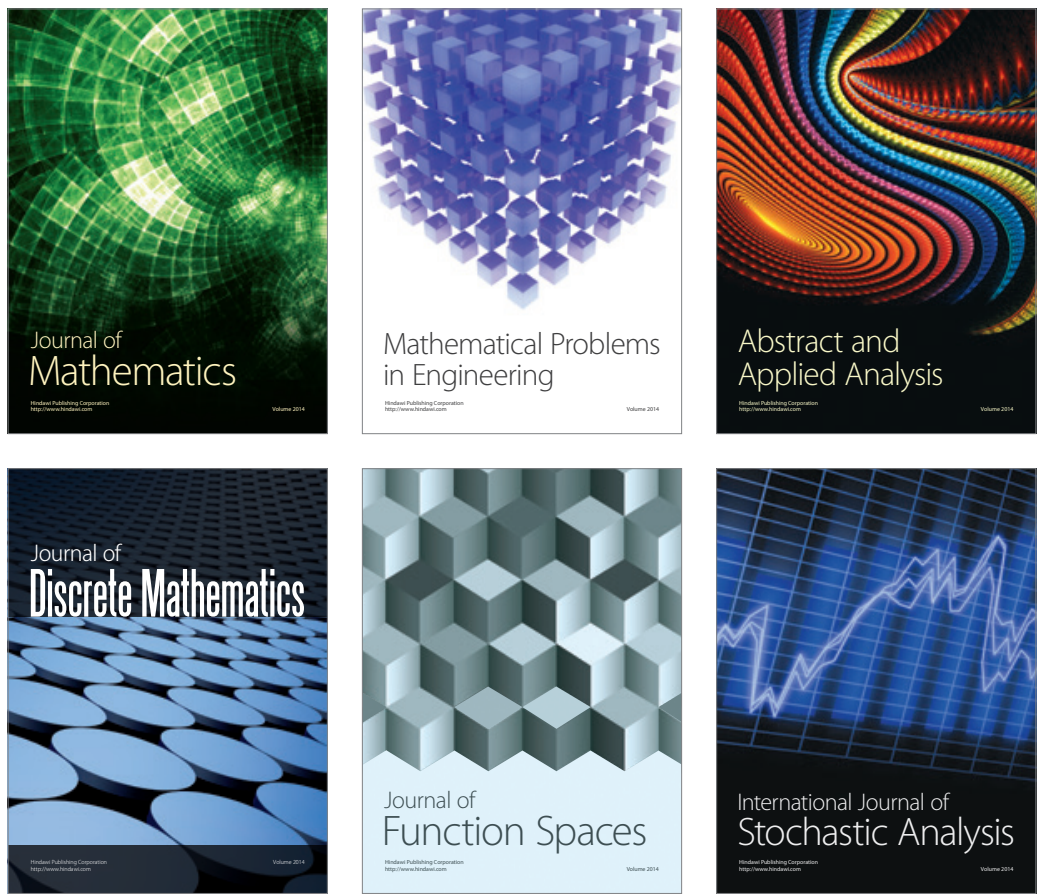

Journal of

Function Spaces

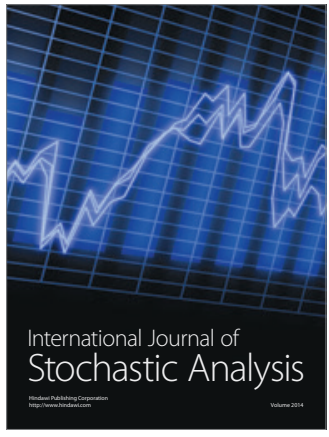

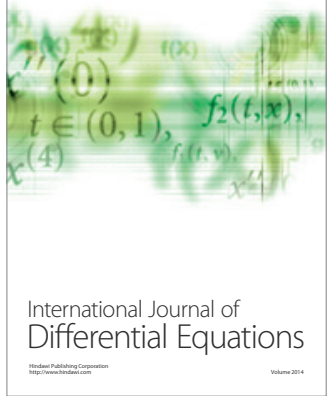
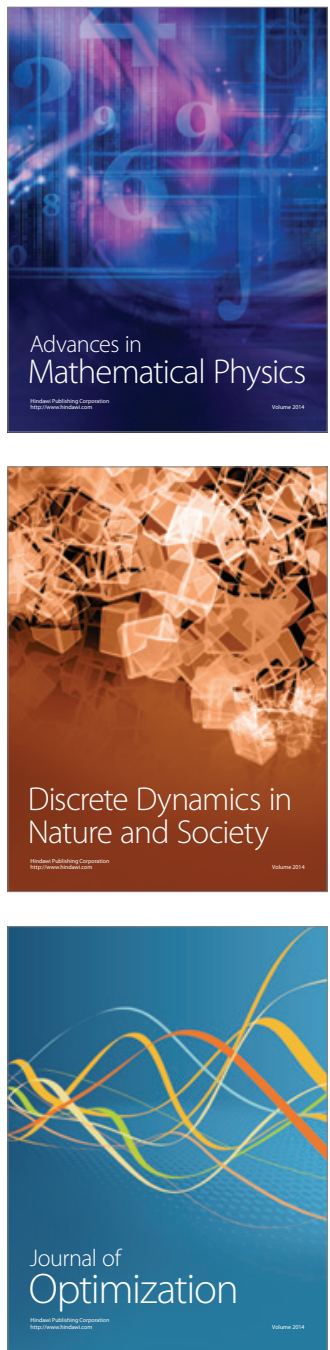